\section{gematik erteilt Zuschlag für neue Kartengeneration}

Der Zuschlag für die Kartengeneration 2 (G2) der elektronischen Gesundheitskarte (eGK) ist am 20.04.2013 erteilt worden. Die zwei identischen Lose der EU-weiten Ausschreibung vom 28. Juli 2012 wurden an Giesecke \& Devrient GmbH (Los 1) und T-Systems International $\mathrm{GmbH}$ (Los 2) vergeben.

Durch den Generationswechsel der eGK werden die vom Bundesamt für Sicherheit in der Informationstechnik (BSI) für die Zukunft empfohlenen noch stärkeren und zugleich langfristig geeigneten kryptographischen Verfahren umgesetzt. Diese Verfahren werden den Datenschutz und die Datensicherheit in der Telematikinfrastruktur weiter erhöhen. Zudem sorgt ein sogenannter Multireferenz-PIN dafür, dass vom Karteninhaber gezielt Bereiche der elektronischen Gesundheitskarte freigeschaltet werden können. Zusätzlich kann die Gültigkeitsdauer der eGK deutlich gesteigert werden.

Der erteilte Auftrag an die Industrie umfasst die Entwicklung eines Kartenbetriebssystems (des sogenannten Card Operating Systems, kurz COS) für die Karten der Generation 2 sowie der Objektsysteme für die Kartentypen elektronische Gesundheitskarte (eGK), Heilberufsausweis (HBA), Institutionskarte (SMC-B) und Gerätekarten (SMC-K, SMC-KT). Die Testkartenlieferung sowie die Lieferung von initialisierten Karten der Generation 2 sind ebenfalls Gegenstand der Beauftragung.

\section{BSI: TR „Ersetzendes Scannen"}

Das Bundesamt für Sicherheit in der Informationstechnik (BSI) hat am 17.04.2013 eine Technische Richtlinie (TR) zum ersetzenden Scannen von Dokumenten (TR-Resiscan-TR 03138) veröffentlicht. Die Technische Richtlinie beschreibt die technischen und organisatorischen Anforderungen für Scanprozesse und -produkte, die erfüllt sein müssen, damit Papierdokumente rechtssicher und gerichtsverwertbar digitalisiert werden können.

Sowohl im behördlichen als auch privat-wirtschaftlichen Umfeld werden zunehmend Dokumente auch in digitalen Dokumentenund Vorgangsbearbeitungs- sowie Aufbewahrungssystemen verarbeitet. Gleichzeitig nimmt das Bedürfnis zu, die Papierdokumente anschließend zu vernichten, um kostenintensive Papierarchive auflösen zu können. Während ein Scanprodukt in rechtlicher Hinsicht nicht denselben Beweiswert wie das originäre Papierdokument haben kann, ist eine Annäherung durchaus möglich. Dies setzt voraus, dass das digitale Endprodukt in einem insbesondere für ein Gericht nachvollziehbaren Scanprozess unter gleichbleibenden qualitativ hochwertigen und abgesicherten Bedingungen entstanden ist. Die dafür notwendigen Anforderungen werden in der Technischen Richtlinie des BSI beschrieben. Die Technische Richtlinie steht auf der Webseite des BSI zur Verfügung: https:// www.bsi.bund.de/DE/Publikationen/TechnischeRichtlinien/tr03138/ index_htm.html

Auf einem gemeinsamen Informationstag des Bundesverbandes IT-Sicherheit e.V. (TeleTrusT) und des BSI wurden am 19.04.2013 in Berlin Inhalte und Anwendungsbeispiele der Richtlinie vor 80 Experten aus Wirtschaft und Verwaltung vorgestellt.

Programm, Referenten und Präsentationen des TeleTrusT-Infor-

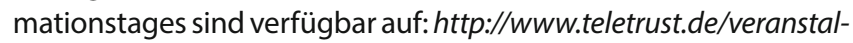
tungen/ersetzendes-scannen/

\section{TeleTrusT-Innovationspreis für IT-Sicherheitslösungen: Bewerbungen bis 31.08.2013}

Der Bundesverband IT-Sicherheit e.V. (TeleTrusT) ruft zu Bewerbungen für den diesjährigen TeleTrusT-Innovationspreis auf. Die Auszeichnung ist für Unternehmen und Institutionen bestimmt, die innovative und vertrauenswürdige IT-Sicherheitslösungen für Wirtschaft und Verwaltung entwickelt haben oder selbst einsetzen. Das Bewerbungsformular ist unter $h t t p: / / w w w . t e l e t r u s t . d e / i n n o v a-$ tionspreis/teletrust-innovationspreis/abrufbar. Bewerbungsschluss ist der 31.08.2013.

Traditionell wird dieser Preis im Rahmen der von TeleTrusT und EEMA ausgerichteten IT-Sicherheitskonferenz „Information Security Solutions Europe" (ISSE; www.teletrust.de/veranstaltungen/isse/) überreicht.

Die Auswahlkriterien für den TeleTrusT-Innovationspreis sind in der Reihenfolge ihrer Gewichtung:

- Grad an Innovation

- Nutzen für die Anwender

- die Konformität mit Standards

- Vorbildcharakter des eingereichten Vorschlages auf nationaler, europäischer und weltweiter Ebene.

Bewertung und Auswahl der Bewerbungen erfolgen durch IT-Sicherheitsexperten. Die Jury steht stellvertretend für den Anspruch von TeleTrusT, IT-Sicherheit interdisziplinär zu betrachten. Die Jury agiert unabhängig von kommerziellen Erwägungen. Die Jury hinterfragt unter anderem, ob der Sicherheitslevel dem Schutzbedarf der Anwendung angemessen ist und ob die Sicherheitsfunktionen integrierter Bestandteil des angebotenen oder genutzten Produktes sind. Besondere Bedeutung wird der Transparenz und Bedienungsfreundlichkeit integrierter Sicherheitsfunktionen beigemessen. Des weiteren wird Wert auf Interoperabilität gelegt sowie darauf, dass für die Anwendung ein messbarer wirtschaftlicher Vorteil nachgewiesen werden kann.

\section{Das DGN ist zertifizierter Anbieter von KV-SafeNet mit Netzkopplung}

Die Kassenärztliche Bundesvereinigung (KBV) hat das Deutsche Gesundheitsnetz zugelassen, KV-SafeNet-Zugänge mit Netzkopplung anzubieten. Die Zertifizierung wurde bereits zum 1. März 2013 erteilt. Somit zählt das DGN zu den ersten Providern, die Kliniken und größere medizinische Einheiten gemäß der KV-SafeNet-Richtlinie (Netzkopplung) 1.0 an das sichere Netz der Kassenärztlichen Vereinigungen anbinden dürfen.

In Krankenhäusern oder Klinikverbünden, in denen mehrere Nutzer standortübergreifend auf das KV-SafeNet zugreifen möchten, muss der herkömmliche Zugang um einen Authentisierungsdienst erweitert werden. Dieser soll eine eindeutige Identifizierung und personenbezogene Authentisierung der Teilnehmer ermöglichen. Das DGN, bereits seit 2005 zertifizierter KV-SafeNet-Provider, hat die neue Richtlinie als integrierte Hard- und Software-Lösung umgesetzt. Als Netzkopplungsrouter dient die speziell für den Klinik-Sektor entwickelte GUSbox $19^{\prime \prime}$.

Der neue Authentisierungsdienst lässt sich per Webbrowser bedienen, so dass die Installation neuer Software entfällt. Die Lösung 